\title{
System-on-Package (SOP) Architectures for compact and low cost RF Front-end modules
}

\author{
S. Pinel, K. Lim, R. G. DeJean, L. Li, C-H.Lee, M. Maeng, M.F. Davis, \\ M. Tentzeris \& J. Laskar.
}

\author{
Packaging Research center, Yamacraw Design Center, School of Electrical and Computer Engineering \\ Georgia Institute of Technology, Atlanta GA 30332-0269 USA \\ Fax:(404) 894-5028, Email; pinel@ece.gatech.edu
}

\begin{abstract}
This paper presents the development of advanced System-on-Package (SOP) architectures for compact and low cost wireless RF wireless systems. We present the design of compact stacked patch antennas using SHS structures for LMDS and Vband applications. Multi-layer organic packaging development for SOP is reported. An Intelligent Network Communicator (INC) RF block is presented as example of the high performances of multi-layer organic package. A novel ultra-compact 3D integration technology for SOP-based solutions is proposed and utilized for the implementation of a Ku band VCO module. In addition, the fabrication of very high $Q$ factor inductors in Liquid Crystal Polymer multi-layer substrate demonstrate superior performances compared to any other multilayer organic packages. I. INTRODUCTION

Multi-layer System-On-Package approach has been demonstrated as capable of overcoming limitation of conventional System-On-Chip approach. It integrates components that would have otherwise been required in discrete form, and MMICs in a single package, hence the term System-on-Package (SOP). In this paper, we present advanced system-on-package architecture development for compact low cost wireless front- end systems. We present the design of compact stacked patch antennas using SHS Structure for LMDS and V-band applications. The multilayer organic packaging development is reported such as an Intelligent Network Communicator (INC), a Universal Information System that integrates digital, optical, analog and RF functions. The implementation of a Ku band VCO module using a novel ultra-compact $3 \mathrm{D}$ integration technology is presented. In addition, the fabrication of very high Q-factor inductors in Liquid Crystal Polymer multi-layer substrate demonstrate superior performances compared to any other multi-layer organic packages.
\end{abstract}

\section{LMDS AND V-BAND ANTENNA DEVELOPMENT USING L TCC BASED P ACKAGE.}

A set of design rules have been established based on LTCC multi-layer technology for the design of compact stacked patch antennas in which only one parameter, the layer thickness, needs to be adjusted in order to achieve an optimal bandwidth performance.
Through simulations using the TLM (transmission line model) based software MicroStripes 5.6, this design strategy has been validated through applying it to LMDS applications at $28 \mathrm{GHz}$. As much as a $-10 \mathrm{~dB}$ return loss bandwidth of 7\% can be achieved for a three-layer LTCC structure (Fig. 1a). Through further adjustments of the antenna dimensions, length and width, as well as feeding the structure along the diagonals of the antenna, circular polarization can be obtained. Finally, to reduce the backside radiation due to surface wave propagation from a high dielectric substrate, the use of a soft-and-hard surface (SHS) [1] has been employed to microstrip patch antennas (Fig. 1b). The SHS is realized by surrounding the patch with rings of metal via rings whose height must be equal to $\lambda_{0} /\left(4^{*} \varepsilon_{\mathrm{r}}{ }^{0.5}\right)$. A reduced backside radiation of approx. 10 $\mathrm{dB}$ is achieved with SHS in comparison to a similar design without SHS at $64.55 \mathrm{GHz}$ (Fig 1c). This design can also achieve a gain enhancement of $10 \mathrm{~dB}$ at broadside (z-direction). The SHS has a broad coverage and a low cross-polarization level [1]. An efficiency of above $85 \%$ is maintained. It has been shown in the past that a large ground plane can also be used to reduce backside radiation, but incorporating the SHS structure to the design can significantly reduce the size of the antenna, therefore making for a more compact module.

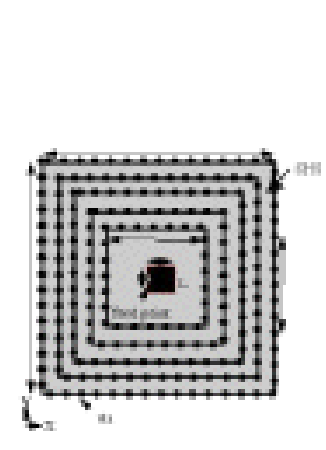

(a)

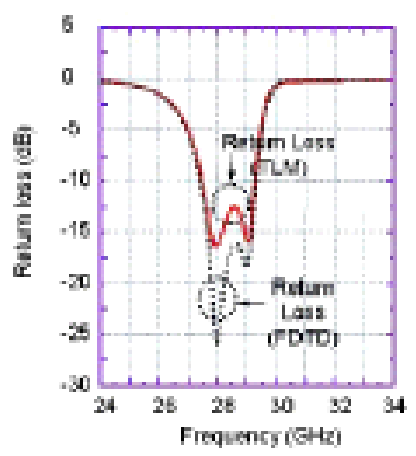

(b) 


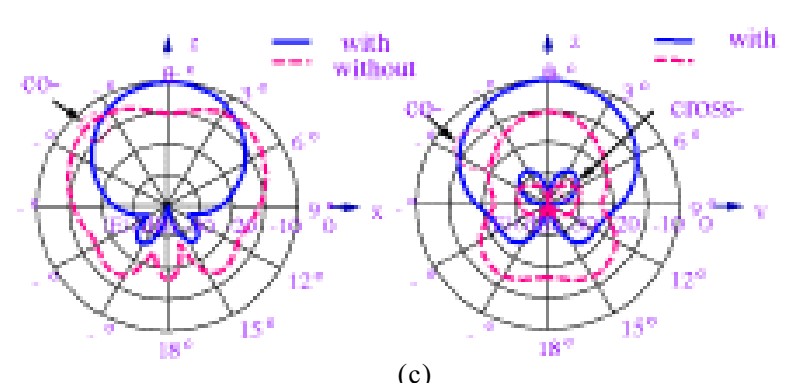

(c)

Fig. 1. SHS structure (a), Return loss characteristic of the stacked patch LMDS antenna (b), Radiation pattern of the stacked patch antenna (c).

\section{AdVAnCED MUlti-laYer ORGANIC BASED PACKAGE.}

\section{A. Intelligent Network Communicator (INC)[2].}

Short distance hybrid digital/RF fiber optic link system (HDROS) is promising because it transmits baseband Ethernet signal and RF signal for GSM/PCS//IMT-2000/WLAN data traffic simultaneously via pre-installed multi-mode fibers. For the implementation of the HDROS, a device, which can combine the digital baseband signal and RF signal with reliable insertion loss and isolation in both band is essential. We developed, in the Multi-Layer Organic (MLO) process, a novel combiner that was optimized for the HDROS and where a $7 \mathrm{~Gb} / \mathrm{s}$ digital baseband signal is simultaneously transmitted with IEEE802.11b WLAN signals up-converted to around $14 \mathrm{GHz}$. Fig 9 shows the basic block of the HDROS.

To overcome the weak coupling at base band and the bandwidth limitation of conventional combiner, we modified the conventional coupled line coupler as vertical coupling structure as shown in Fig 10. The output port of the coupled line coupler is used as the input port for the RF signal and the isolation port is shorted to the ground. Also we designed 9th order Bessel LPF at the input port for the base band, which performs as band stop filter for the $14 \mathrm{GHz}$ RF signal and reflects the RF signal at the output of the LPF. We optimized the $14 \mathrm{GHz}$ RF signal in-phase to obtain a constructive effect at the output port of the combiner by adjusting the embedded microstrip line which interconnects the LPF with vertical coupling structure. The design was finalized with the $3 \mathrm{D}$ method of momentum electromagnetic simulation software.

We used the HP8510 Vector Network Analyzer (VNA) to measure the frequency characteristic of the combiner. The insertion loss of the RF signal at the output port of the combiner was measured as $1.9 \mathrm{~dB}$. The isolation between port 1 and port 2 of the combiner was greater than $10 \mathrm{~dB}$ in base band and $38 \mathrm{~dB}$ at $14 \mathrm{GHz}$. These results satisfy the requirement for the proposed HDROS. $3 \mathrm{~dB}$ bandwidth of $7 \mathrm{GHz}$ is achieved between port 1 and port 3. Fig. 11 shows the measured frequency spectrum at the output of the combiner when $7 \mathrm{~Gb} / \mathrm{s}$ PRBS with $14 \mathrm{GHz}$ sinusoidal wave are sent. Fig. 11 also shows the measured eye opening of the $7 \mathrm{~Gb} / \mathrm{s}$ PRBS at the output port.

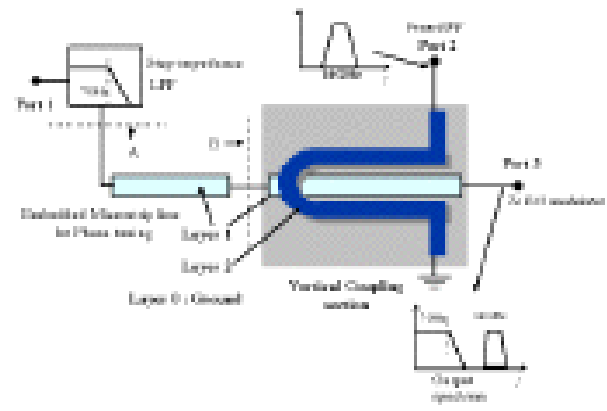

Fig. 9 Schematic configuration of INC.

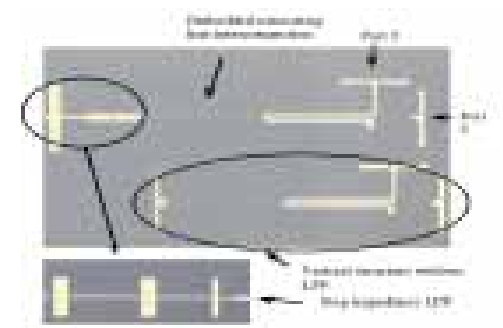

Fig. 10 Picture of fabricated combiner in MLO process

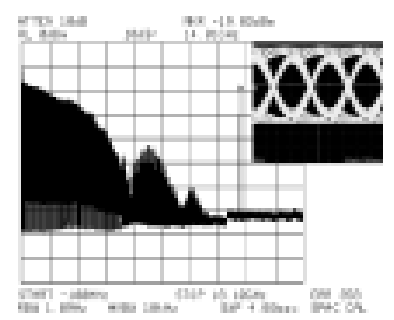

Fig. 11 Frequency spectrum at the output port of the combiner and eye diagram

\section{B. Ultra-compact Ku-Band VCO Module [3].}

The proposed module concept is illustrated in Fig.3a. A microwave multi-layer interconnect structure is built on a glass carrier using modified MCM-D technology and advanced photosensitive epoxy. Low loss interconnect is fabricated using build-up technology and $12 \mu \mathrm{m}$ thick electroplated copper. Micro-via technology with minimum diameter of $40 \mu \mathrm{m}$ is used to connect the different metal layers. Thus high density interconnect network and integral passive components such as high performances embedded inductors, filters and antennas can be implemented within the multi-layer wiring structure RF commercial chipset are directly embedded into the MCM-D structure. Furthermore, glass carrier substrate 
used during the fabrication can be selectively etched and removed, leading to a final thickness of only 150 microns. We fabricated coplanar wave-guides (CPW) and test structures dedicated to vertical via characterization. A CPW line of a length of 25 mils exhibits measured performances such as $\mathrm{S}_{11}<-35 \mathrm{~dB} @ 14 \mathrm{GHz}$ and $\mathrm{S}_{21}=$ $0.018 \mathrm{~dB} @ 14 \mathrm{GHz}$. A CPW-to-CPW transition by means of vertical micro-via exhibits measured performances such as $\mathrm{S}_{11}<-20 \mathrm{~dB} @ 14 \mathrm{GHz}$ and $\mathrm{S}_{21}=-0.04 \mathrm{~dB} @ 14 \mathrm{GHz}$.

Also, we fabricated high Q RF inductors (Fig 3b). Particular care was been given to perform very accurate LRRM calibration for high Q measurements, and hybrid parameters values have been extracted directly from the measured data. The fabricated inductor exhibits inductance values ranging from 0.4 up to $2 \mathrm{nH}$, quality factor up to 80 and self-resonance frequency from $12 \mathrm{GHz}$ to $37 \mathrm{GHz}$ as shown in table 1.

TABLE I

SUMMARY OF MEASURED RF INDUCTORS PERFORMANCES.

\begin{tabular}{|c|c|c|}
\hline $\begin{array}{c}\mathbf{L} \\
\mathbf{n H}\end{array}$ & $\begin{array}{c}\text { Quality } \\
\text { Factor }\end{array}$ & $\begin{array}{c}\text { SRF } \\
\mathbf{G H z}\end{array}$ \\
\hline $\mathbf{0 . 4}$ & $\mathbf{8 0} @ 10 \mathrm{GHz}$ & $\mathbf{3 7}$ \\
\hline $\mathbf{0 . 5}$ & $\mathbf{8 0} @ 8 \mathrm{GHz}$ & $\mathbf{3 2}$ \\
\hline $\mathbf{2}$ & $\mathbf{4 0} @ 4 \mathrm{GHz}$ & $\mathbf{1 2}$ \\
\hline
\end{tabular}

A Ku-band VCO (Fig 3b) has been implemented in a $0.125-\mu \mathrm{m} \mathrm{GaAs}$ pHEMT process and occupies an area of $0.62 \times 0.55 \mathrm{~mm}^{2}$. A common gate configuration is used to generate strong negative resistance by inductive feedback. The resonator of the VCO consists of a spiral inductor, MIM capacitors, and a diode, which is used for frequency tuning. To eliminate undesirable low frequency oscillations, an LC network is also incorporated as a high pass filter on the output path.

On-wafer measurements of oscillation frequency, output power, and phase noise were performed using an Agilent8563E spectrum analyzer. A maximum output power of $10.67 \mathrm{dBm}$ was measured. The signal spectrum of the VCO over a $10 \mathrm{MHz}$ span has been measured and showed a phase noise of $-101.8 \mathrm{dBc} / \mathrm{Hz}$ at a $1 \mathrm{MHz}$ offset. After the embedding process and before the last metalization, the measured oscillation frequency is 16 $\mathrm{GHz}$. The $300 \mathrm{MHz}$ offset with the bared die performances is attributed to the impact of the dielectric on the on-chip passives components determining the gate load which is critical in determining the conditions of oscillation. Fig. $3 \mathrm{c}$ shows the output spectrum for the embedded VCO after the last metalization (cf. Fig.3a). The measured oscillation frequency is $15.4 \mathrm{GHz}$. The $600 \mathrm{MHz}$ additional offset is attributed to the impact of the metal traces on reflection coefficient at the RF-output port. The maximum output power of $10.67 \mathrm{dBm}$ and the phase noise of $-101.8 \mathrm{dBc} / \mathrm{Hz}$ at a $1 \mathrm{MHz}$ offset remain unchanged .

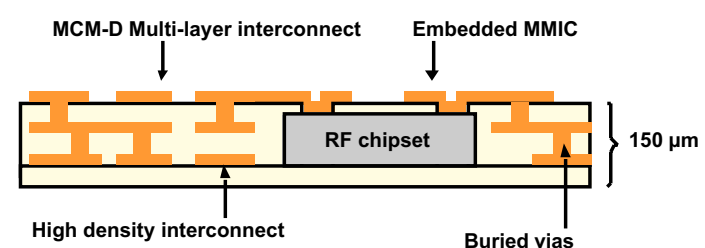

Fig. 3a. Concept view of Ultra-compact module.

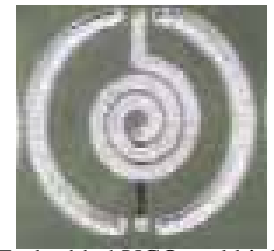

Fig. 3b. Embedded VCO and high Q RF inductors.

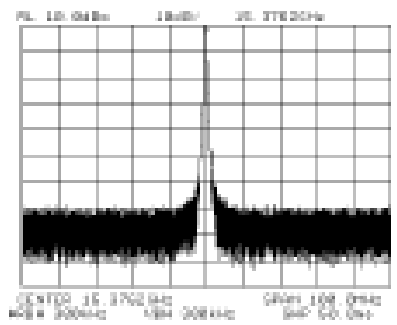

Fig. 3c. Frequency spectrum at the output port.

\section{High Q RF passives using Liquid Crystal Polymer} multi-layer substrate [4].

LCP (Liquid Crystal Polymer) is a fairly new and promising thermoplastic material. It can be used as a low cost dielectric material or, single sheeted or laminated, as a substrate for high volume large area processing methods that provides very reliable high performances circuits at low cost. LCP have a unique combination of properties that makes it ideally suited for high-density high-speed digital and millimeter wave multi-layer substrate applications. These performances are better than any other organics based material currently used in PWB fabrication and can be quite comparable to ceramic-based substrate widely used for RF and millimeter waves applications.

LCP properties include:

- Excellent electrical properties up to millimeter waves, such as low dielectric constant (2.9@20 GHz) and low dielectric losses (Loss tangent of $0.002 @ 20$ $\mathrm{GHz}$ ) comparable with ceramics.

-Very good barrier properties, permeability comparable to that of glass and very close to that of ceramic. LCP is virtually impermeable to oxygen and other gases and liquids. LCP has very low moisture absorption $(\sim 0.02 \%) 50$ to 100 times less than polyimide and epoxy films which leads to a better stability.

- Low Coefficient of Thermal Expansion (CTE) as low as $8 \cdot 10-6 / \mathrm{K}$, adjustable through thermal treatments process coefficient of thermal expansion, and close to that 
of ceramic $(6 \cdot 10-6 / \mathrm{K})$ (cf. table 2$)$. This leads to higher matching to silicon or chip package and provides higher reliability than board made from FR4 or polyimide. LCP is also manufactured with different glass transition temperature $\mathrm{Tg}$ from $280^{\circ} \mathrm{C}$ to well over $350^{\circ} \mathrm{C}$.

Material, electrical and economical considerations make LCP a serious candidate for all Multi-Chip-Module (MCM), Systems-On-Package (SOP) and advanced packaging technology lead by the tremendous growing market for Digital, RF and Opto-RF applications.

A complete library of multi-layer circular inductors was fabricated using LCP and measured (Fig 4b). The fabricated inductors exhibit inductance values ranging from 1.1 up to $4 \mathrm{nH}$, quality factor up to 90 and self-resonance frequency from $8 \mathrm{GHz}$ to $16 \mathrm{GHz}$ (table 2).

TABLE II

COMPARISON OF SUBSTRATE PROPERTIES.

\begin{tabular}{|c|c|c|}
\hline $\begin{array}{c}\text { L } \\
\mathbf{n H}\end{array}$ & $\begin{array}{c}\text { Quality } \\
\text { Factor }\end{array}$ & $\begin{array}{c}\text { SRF } \\
\text { GHz }\end{array}$ \\
\hline $\mathbf{1 . 1}$ & $\mathbf{9 0} @ 5.8 \mathrm{GHz}$ & $\mathbf{1 6}$ \\
\hline $\mathbf{1 . 5}$ & $\mathbf{9 0} @ 4 \mathrm{GHz}$ & $\mathbf{1 5}$ \\
\hline $\mathbf{2}$ & $\mathbf{8 0} @ 4 \mathrm{GHz}$ & $\mathbf{1 5}$ \\
\hline $\mathbf{4}$ & $\mathbf{7 0} @ 2.4 \mathrm{Ghz}$ & $\mathbf{8}$ \\
\hline
\end{tabular}

Table 2: summary of measured RF inductors performances on LCP.

The physical based model we developed initially did not model accurately the degradation of the quality factor due to radiation losses. Therefore, polynomial frequency dependency was added to the expression of the series resistance Rs in order to model radiations losses and lead to better agreement with measurements for Q@5.8Ghz about 90. The total series resistance expression Rrad is described in equation 1 and figure 4a shows measurements results compared with the model.

$$
\begin{aligned}
& R \operatorname{rad}=R s \cdot\left[1+0.1\left(\frac{f}{f c}\right)^{5}\right] \\
& \text { with } f c=8 \mathrm{GHz}
\end{aligned}
$$

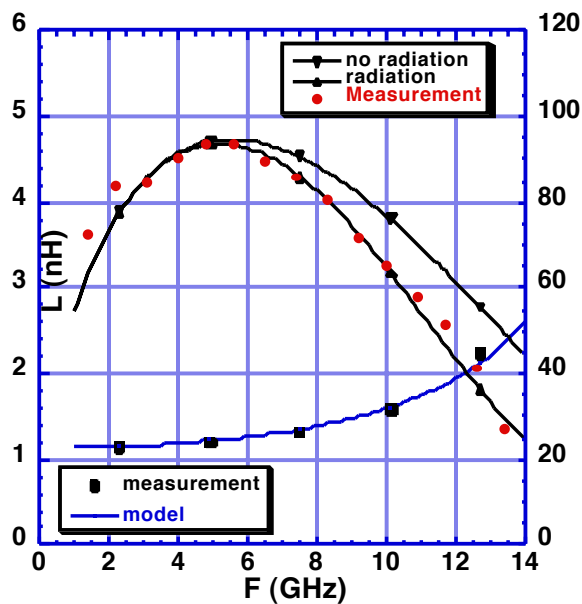

Fig. 4a. Measurement performances of High Q RF inductors.

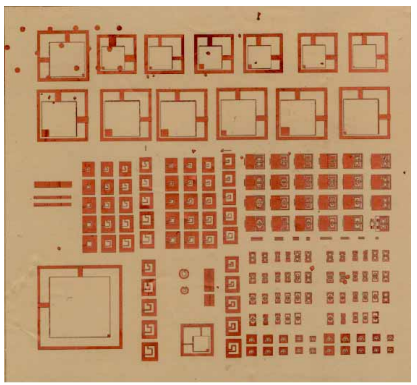

Fig. 4b. LCP test coupon

\section{CONCLUSION}

We have presented advanced System-on-Package (SOP) architectures for compact and low cost wireless RF wireless systems. The design of compact stacked patch antennas using SHS Structure for LMDS and V-band applications. Multi-layer organic packaging developed for SOP is reported. An Intelligent Network communicator (INC) RF block have been presented as examples of the high performances of multi-layer organic package. The implementation of a $\mathrm{Ku}$ band VCO module using a novel ultra-compact 3D integration technology is proposed. In addition, the fabrication of very high Q-factor inductors in Liquid Crystal Polymer multi-layer substrate demonstrate superior performances compared to any other multi-layer organic packages.

\section{A CKNOWLEDGEMENT}

The authors wish to acknowledge ASAHI Inc, Triquint Semiconductor, the Packaging Research Center of Georgia-Tech, for their technical support.

\section{REFERENCES}

[1] Kildal, P.-S., "Artificially soft and hard surfaces in electromangetics,", IEEE Trans. Antennas Propagat., vol. 38, no.10, 1990, pp. 1537-1544.

[2] Maeng, M.; Lim, K.; Hur, Y.; Davis, M.; Lal, N.; Yoon, S.W.; Laskar, J.; "Novel combiner for hybrid digital/rf fiberoptic application", Radio and Wireless Conference, 2002. RAWCON 2002 IEEE, 2002 Page(s):193-1

[3] S. Pinel, CH. Lee, S-W. Yoon, K.Lim, and J. Laskar., "Embedded IC packaging technology for ultra-thin and highly 훈 compact RF module", ECTC 2003, New Orleans, 27-30 Mai 2003, pp 24-29.

[4] S. Pinel, M.Roellig, R.Kunze, V.Sundaram, K.Lim, J.Laskar, G.White and R.Tummala, "Stacked Liquid Crystal Polymer substrates and $\mu \mathrm{BGA}$ technology for 3D integrated C-band RF Front-End Module", $A P M C$ 2002, Kyoto, Novembre 2002, pp 955-958. 